Original Research Paper

\title{
Multivariate analysis of body weight, morphometric and thermo-physiological traits of indigenous pigs under tropical conditions
}

\author{
Yakubu, A. ${ }^{*}$, Durven, G.L. ${ }^{1}$, Hagan, J. ${ }^{2}$ \\ ${ }^{I}$ Department of Animal Science, Faculty of Agriculture, Nasarawa State University, Keffi, Shabu-Lafia \\ Campus, P.M.B. 135, Lafia, Nasarawa State, Nigeria. \\ ${ }^{2}$ Department of Animal Science, School of Agriculture, University of Cape Coast, \\ Ghana
}

*Corresponding Author: Abdulmojeed Yakubu ,Lafia, Nasarawa State, Nigeria

Email: abdulmojyak@gmail.com Mobile No: +2348064654748

Running title: Multivariate characterization of indigenous pigs

Article history; Received: 03.07.2021; Revised: 27.08.2021; Accepted: 01.01.2022

\begin{abstract}
The study aimed at describing objectively the interdependence among the morphological and heat tolerance traits of Nigerian indigenous pigs and to predict body weight from conformation traits. Data on body weight, eight linear body measurements (BL, CG, CD, WH, RH, EL, SL and TL) and three thermo-physiological parameters were measured on 150 randomly selected pigs of three growth stages (piglets, growers and finishers) from February to December, 2020. The animals were managed in an extensive system in Plateau State, north central Nigeria. General linear model was used to study growth stage and sex effects including their interaction. Multivariate principal component analysis was used for the size, shape and heat tolerance determination while the animals were classified using canonical discriminant analysis. The stepwise regression was used for body weight prediction. The results showed that finishers had significantly higher $(\mathrm{P}<0.05)$ body weight, body length, chest girth, chest depth, withers height, rump height, ear length, snout length and tail length, followed by growers while the least values were recorded in piglets. Pulse rate was not significantly different $(\mathrm{P}>0.05)$ between piglets and finishers, although the latter had higher respiratory rate $(39.48 \pm 0.53$ vs. $39.90 \pm 0.53$ vs. $36.77 \pm 0.75)$. However, rectal temperature was similar $(\mathrm{P}>0.05)$ among the three pig categories. With the exception of tail length, sexual dimorphis $\mathrm{m}$ was observed in all the morphometric traits with higher values recorded for males. However, the three thermo-physiological traits were not affected by sex $(\mathrm{P}>0.05)$. BW was highly and positively correlated with most biometric traits $(\mathrm{r}=0.80-$ 0.93, 0.66-0.80 and 0.83-0.93; $\mathrm{P}<0.01$ for piglets, growers and finishers, respectively). Three principal components (PC1, PC2 and PC3) were extracted for optimal balance of the animals. Withers height, ear length and body weight were found to be the most discriminating variables to separate the pig categories. Classification results showed that $100 \%$ of piglets, $96.7 \%$ of growers and $96.7 \%$ of finishers were correctly as signed to their distinct populations. The present Information could be exploited in devising appropriate management and breeding programs for tropically adapted pigs in Nigeria.
\end{abstract}

Keywords: Nigerian indigenous pigs, conformation, measurements, heat tolerance, multivariate analysis 


\section{Introduction}

Pigs are one of the most prolific and fast growing livestock that can convert feed and food waste to valuable products. The value of a pig carcass for meat production depends primarily on carcass weight and the relation proportions of fat and lean meat (Brown-Brandi et al., 2004). Indigenous breeds are usually owned by rural farmers, although they may not yield as much in production as the exotic breeds. They produce a wider range of products, thrive on low forage and require lower levels of health care. Their management is ecologically more sustainable, especially in marginal environments (KohlerRollefson, 2000; Rege et al., 2011). According to the 2011 National Agricultural Sample Survey, the population of pigs in Nigeria was put at 7.1 million compared to an estimated 19.5 million goats and 41.3 million sheep (NBS, 2011). Despite decreasing trends in population size, the existing indigenous pigs still represent a valuable component of local animal genetic resources. Evaluating and assessing the phenotypic variation among native pig populations are important to identify the uniqueness of populations and possible gene flow between indigenous pigs.

External appearance (morphology) is still commonly used by researchers and practitioners in the identification, characterization and selection of farm animals (Yakubu, 2013; Shi et al., 2020; Siewe et al., 2021; Zhang et al., 2021). . Alternative body measurements and indices estimated from different combinations of different body traits produced a superior guide to weight and were also used as an indicator of type and function in domestic animals (Schwabe and Hall, 1989; Fernandes et al., 2019). Morenikeji et al. (2019) have used linear body measurements such as body length, trunk length, height at withers, chest girth, tail length and shoulder to tail length to characterize pigs. In a related study, Al Ard Khanji et al. (2018) used flank-to-flank distance, heart girth, length and loin depth to predict body weight. Also, Panda et al. (2021) reported that body length, heart girth, paunch girth, height at wither, height at back, rump width, thigh circumference, neck circumference, and body depth had high correlation coefficients $(0.8-0.97)$ with body weight $(\mathrm{BW})$ at both weaning and post-weaning stages; thereby permitting the estimation of BW from the former.

Changes in thermo-physiological traits such as rectal temperature, respiratory rate and pulse rate can be used to evaluate the capacity of adaptation of pigs to hot-dry conditions. The understanding of the animal responses to thermal challenge is paramount to successful implementation of breeding strategies to increase production and productivity of pigs under cold and warm climates (Sipos et al., 2013).

A considerable number of techniques describing pig morphometric have been utilized in the past (Muta $e t$ al., 2011; Adeola et al., 2013; Walugembe et al., 2014). However, multivariate principal component and discriminant analyses, which have been successfully used in other livestock species (Yakubu et al., 2010; Fraga et al., 2016; Belkhadem et al., 2019; Mediouni et al., 2020; Meka et al., 2021; Abed et al., 2021) could be invaluable refined techniques for obtaining very useful information related to several characteristics of pigs (Ventura et al., 2012) including body measurements (Silva Filha et al., 2010) and thermo physiological traits. Evaluating and assessing the body weight, morphometric and heat-tolerance traits of animals are important to identify the uniqueness of populations, adaptive capacity and possible gene flow between the indigenous populations. In north central Nigeria, there is dearth of information on body size, conformation and heat stress parameters to inform decisions on breeding and management of pigs especially under the low-input, smallholder production systems. The present study, therefore, aimed at determining the body weight, morphometric characteristics and thermo-physiological traits of indigenous pigs in Nigeria using multivariate techniques.

\section{Materials and Methods}

\section{Study Area}

The study was conducted in Plateau State, north central Nigeria. Plateau State is located between latitude $80^{\circ} 24^{\prime}$ North and longitude $80^{\circ} 32^{\prime}$ and $100^{\circ} 38$ east. The altitude ranges from 1,200 meters (400 feet) to a peak of 1,829 meters above sea level in the Shere Hills range near Jos. 


\section{Sampling of animals}

A total number of one hundred and fifty (150) indigenous pigs of different physiological ages were randomly sampled from the 3 agricultural zones (Plateau North, Plateau Central and Plateau South) of Plateau State, Nigeria. In each of the 3 agricultural zones, 50 pigs (of both sexes) were considered (20 adults, 20 growers and 10 piglets, respectively). They were further classified as 0-6 weeks (piglets), 7-13 weeks (growers) and 14-24 weeks (Finishers). The study was carried out from February to December, 2020.

\section{Morphometric Data}

- Body weight $(\mathrm{kg})$ (measured with a digital scale) and the following eight linear body measurements $(\mathrm{cm})$ were taken using the anatomical measurements described by ESGPIP (2009) and AU-IBAR (2015):

- Body length (BL): This is the length from the base of the tail to the middle of the shoulder blade along the back on the middle line or the distance from the base of the ear to the base of the tail. A graduated meter rule was used

- Chest girth (CG): The chest girth was measured around the chest just behind the front legs and withers.

- Withers height (WH): This is the distance from the surface of a platform on which the animal stands to the withers, or midpoint of the shoulder blade to the floor in the perpendicular plane. A graduated metre rule was used in the measurement.

- Rump height (RH): Rump height is the distance from the surface of a platform to the rump, using a meter rule.

- Ear length (EL): The distance between the tip of the ear and the base was taken using a measuring tape.

- Snout length (SL): The distance between the frontal nasal suture and upper part of the snout.

- Chest depth (CD): The distance from the back bone at the shoulder (standardized on one of the vertical processes of the thoracic vertebrae) to the brisket between the front leg.

- Tail length (TL): The length of the tail from the base of its attachment to the tip.

\section{Thermo-physiological data}

Pulse rate (PR): This was examined at the coccygeal artery, or at the femoral artery by counting the pulse waves per minute (bpm) using a stethoscope of normal range value of 90-110 bpm.

Respiratory rate (RR): This was determined by counting respiratory movement of the thorax per minute (bpm) using the finger tip.

Rectal temperature (RT): This was determined by the use of a clean digital thermometer $\left(38.5-40^{\circ} \mathrm{C}\right)$.

\section{Statistical analysis}

Data were analyzed using the general linear model (GLM) procedure of SPSS (2017) software to test the fixed effects of growth stage and sex as well as their interaction on BW, BL, CG, CD, WH, RH, EL, SL, TL, RR, PR and RT. Means were separated using the least significant difference (LSD) method at $95 \%$ confidence interval. The following linear model was employed:

$Y_{i j k}=$ individual observation

$$
\mathbf{Y}_{\mathrm{ijk}}=\boldsymbol{\mu}+\mathbf{G}_{\mathrm{i}}+\mathrm{S}_{\mathrm{j}}+(\mathbf{G S})_{\mathrm{ij}}+\mathbf{e}_{\mathrm{ijk}}
$$

$\mu=$ overall mean

$G_{i}=$ fixed effect of $i^{\text {th }}$ growth stage $(i=$ piglets, growers and finishers $)$.

$S_{j}=$ fixed effect of $j^{\text {th }} \operatorname{sex}(j=$ male, female $)$

$(G S)_{i j}=$ interaction effect of $i^{\text {th }}$ growth stage and $j^{\text {th }}$ sex

$e_{i j k}=$ random error associated with each record (normally, independently and identically distributed with zero mean and constant variance) 
Pearson's coefficients of correlations were computed for all the traits. The multivariate principal component (PC) and discriminant analyses were also employed. Among the many multivariate analysis methods, principal component analysis is a simple powerful one that has been advocated for analysis of population genetic relationship (Vohra et al., 2015). The objective of PC analysis is to account for the maximum numbers of composite variables. The criterion of eigenvalues greater than 1 (one) was used to retain the main components. The reliability of the PC analysis was tested using anti-image correlations, The Kaiser Meyer Olkin measure of sampling adequacy and Bartlett Test of Sphericity.

In order to identify the combination of variables that best separate the three growth stages, canonical discriminant analysis was used (Tabachnick and Fidel, 2001). The ability of this discriminant model to identify piglets, growers and finishers was indicated as the percentage of individuals correctly assigned to its a priori group. Split-sample validation (cross-validation) was used to evaluate the accuracy of the classification.

A multiple regression procedure using a stepwise variable selection was used to obtain models of estimation of BW from biometric measurements. SPSS (2017) was used in all analyses.

\section{Results}

The body weight, linear body measurements and heat tolerance characteristics of piglets, growers and finishers are presented in Table 1. The results revealed that finishers had significantly higher $(\mathrm{P}<0.05)$ body weight, body length, Chest girth, Chest depth, withers height, rump height, ear length, snout length and tail length, followed by growers while the least values were recorded in piglets. Pulse rate was not significantly different $(\mathrm{P}>0.05)$ between piglets and finishers, although the latter had higher respiratory rate $(39.48 \pm 0.53$ vs. $39.90 \pm 0.53$ vs. $36.77 \pm 0.75)$. However, rectal temperature was similar $(\mathrm{P}>0.05)$ among the three pig categories.

Component Plot in Rotated Space

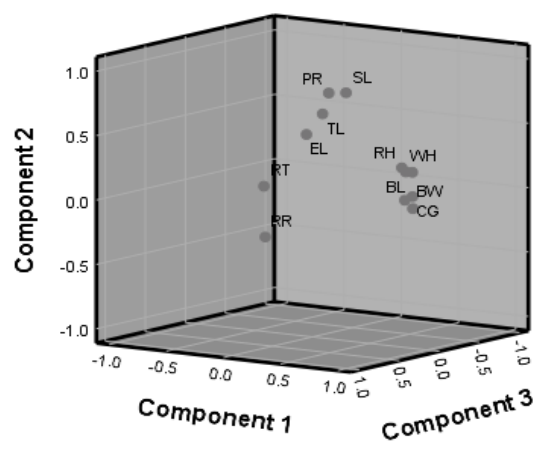

Figure 1. The three principal components plot in rotated space.

With the exception of tail length $(\mathrm{P}>0.05)$, body weight and linear body measurements were significantly higher $(\mathrm{P}<0.05)$ in males compared to their female counterparts (Table 2). However, rectal temperature, pulse rate and respiratory rate were not significantly influenced $(\mathrm{P}>0.05)$ by sex.

There was significant $(\mathrm{P}<0.05)$ growth stage and sex interaction effect on $\mathrm{BW}, \mathrm{BL}, \mathrm{CG}, \mathrm{CD}, \mathrm{WH}, \mathrm{RH}$ and RR. Male finishers had significantly higher BW (29.83 vs. 22.17; $\mathrm{P}<0.05)$ compared to females, whereas BW in both sexes was statistically similar $(\mathrm{P}>0.05)$ among piglets and growers (Table 3$)$. Male finishers also had higher BL (47.63 vs.38.90), CG (51.37 vs. 41.43), WH (43.37 vs. 39.50) and RH (45.13 vs. 
41.18; $\mathrm{P}<0.05)$. The $\mathrm{CD}$ also favoured $(\mathrm{P}<0.05)$ male growers $(16.39$ vs. 14.75$)$ and finishers $(26.63$ vs. $22.63)$, respectively. With respect to $R R$, however, significantly higher $P<0.05$ value was found in females (39.13 vs. 34.40).

Table 1. Body weight, conformation and heat tolerance traits (Means \pm SE) of different categories of pigs in Plateau State

\begin{tabular}{llll} 
& \multicolumn{3}{c}{ Growth Stage } \\
\cline { 2 - 4 } Parameters & Piglets & Growers & Finishers \\
\hline Body weight & $6.60 \pm 0.78^{\mathrm{c}}$ & $12.35 \pm 0.54^{\mathrm{b}}$ & $26.00 \pm 0.54^{\mathrm{a}}$ \\
\hline Body length & $22.45 \pm 0.91^{\mathrm{c}}$ & $29.77 \pm 0.64^{\mathrm{b}}$ & $43.27 \pm 0.64^{\mathrm{a}}$ \\
\hline Chest girth & $17.88 \pm 1.03^{\mathrm{c}}$ & $26.88 \pm 0.73^{\mathrm{b}}$ & $46.40 \pm 0.73^{\mathrm{a}}$ \\
\hline Chest depth & $8.02 \pm 0.53^{\mathrm{c}}$ & $15.57 \pm 0.37^{\mathrm{b}}$ & $24.63 \pm 0.37^{\mathrm{a}}$ \\
\hline Withers height & $19.98 \pm 0.47^{\mathrm{c}}$ & $29.02 \pm 0.33^{\mathrm{b}}$ & $41.43 \pm 0.33^{\mathrm{a}}$ \\
\hline Rump height & $20.64 \pm 0.51^{\mathrm{c}}$ & $30.07 \pm 0.36^{\mathrm{b}}$ & $43.16 \pm 0.36^{\mathrm{a}}$ \\
\hline Ear length & $2.87 \pm 0.20^{\mathrm{c}}$ & $7.59 \pm 0.14^{\mathrm{b}}$ & $10.82 \pm 0.14^{\mathrm{a}}$ \\
\hline Snout length & $3.81 \pm 0.32^{\mathrm{c}}$ & $8.6 \pm 0.23^{\mathrm{b}}$ & $12.12 \pm 0.23^{\mathrm{a}}$ \\
\hline Tail length & $3.74 \pm 0.34^{\mathrm{c}}$ & $8.14 \pm 0.24^{\mathrm{b}}$ & $10.70 \pm 0.24^{\mathrm{a}}$ \\
\hline Rectal temperature & $39.32 \pm 0.53^{\mathrm{a}}$ & $38.47 \pm 0.37^{\mathrm{a}}$ & $37.93 \pm 0.37^{\mathrm{a}}$ \\
\hline Pulse rate & $78.73 \pm 1.67^{\mathrm{a}}$ & $75.32 \pm 1.18^{\mathrm{b}}$ & $79.93 \pm 1.18^{\mathrm{a}}$ \\
\hline Respiratory rate & $36.77 \pm 0.75^{\mathrm{b}}$ & $39.90 \pm 0.53^{\mathrm{a}}$ & $39.48 \pm 0.53^{\mathrm{a}}$
\end{tabular}

S.E. = Standard error of means

${ }^{a b c}$ Means within rows carrying different superscripts are significantly different $(P<0.05)$

Table 2. Effect of sex on body weight, conformation and heat tolerance traits (Means \pm SE) of pigs

\begin{tabular}{lll} 
& \multicolumn{2}{c}{ Sex } \\
\cline { 2 - 3 } Parameters & Female & Male \\
\hline Body weight & $13.11 \pm 0.51^{\mathrm{b}}$ & $16.84 \pm 0.51^{\mathrm{a}}$ \\
\hline Body length & $30.01 \pm 0.60^{\mathrm{b}}$ & $33.65 \pm 0.60^{\mathrm{a}}$ \\
\hline Chest girth & $28.21 \pm 0.69^{\mathrm{b}}$ & $32.56 \pm 0.69^{\mathrm{a}}$ \\
\hline Chest depth & $14.93 \pm 0.35^{\mathrm{b}}$ & $17.21 \pm 0.35^{\mathrm{a}}$ \\
\hline Withers height & $29.07 \pm 0.31^{\mathrm{b}}$ & $31.22 \pm 0.31^{\mathrm{a}}$ \\
\hline Rump height & $30.30 \pm 0.34^{\mathrm{b}}$ & $32.27 \pm 0.34^{\mathrm{a}}$ \\
\hline Ear length & $6.81 \pm 0.14^{\mathrm{b}}$ & $7.37 \pm 0.14^{\mathrm{a}}$ \\
\hline Snout length & $7.78 \pm 0.21^{\mathrm{b}}$ & $8.58 \pm 0.21^{\mathrm{a}}$ \\
\hline Tail length & $7.44 \pm 0.23^{\mathrm{a}}$ & $7.61 \pm 0.23^{\mathrm{a}}$ \\
\hline Rectal temperature & $38.80 \pm 0.35^{\mathrm{a}}$ & $38.34 \pm 0.35^{\mathrm{a}}$ \\
\hline Pulse rate & $77.51 \pm 1.12^{\mathrm{a}}$ & $78.48 \pm 1.12^{\mathrm{a}}$ \\
\hline Respiratory rate & $39.30 \pm 0.50^{\mathrm{a}}$ & $38.13 \pm 0.50^{\mathrm{a}}$ \\
\hline
\end{tabular}

In piglets, while BW was highly and positively correlated with $\mathrm{WH}, \mathrm{CG}, \mathrm{CD}, \mathrm{SL}, \mathrm{RH}, \mathrm{TL}, \mathrm{EL}$ and $\mathrm{BL}$ (r $=0.93,0.92,0.91,0.89,0.88,0.88,0.85,0.80 ; \mathrm{P}<0.01)$, it was negatively associated with RT $(-0.66)$ (Table 4). Incidentally, RT was positively and negatively correlated with all the body conformation traits $(\mathrm{r}=-0.66-0.95 ; \mathrm{P}<0.01)$ while $\mathrm{PR}$ was related to $\mathrm{BL}$ and $\mathrm{CG}(\mathrm{R}=-0.68$ and $-0.67 ; \mathrm{P}<0.01)$. However, the highest correlation coefficient was found between WH and RH $(\mathrm{r}=0.98 ; \mathrm{P}<0.01)$ while the lowest was between CG and RR ( $r=0.02 ; \mathrm{P}>0.05)$. Among growers, $\mathrm{BW}$ was also highly and significantly $(\mathrm{P}<0.01)$ related to WH (0.80), BL (0.77), CG (0.72), EL (0.68) and RH (0.66). In this group, statistically significant positive correlation was observed between BW and RT (0.26), the latter was also positively correlated with CG (0.41) and BL (0.34). PR was negatively related to CD (-0.65), TL (-0.58), SL (-0.48) and EL (-0.41) while RR had positive relationship with CD (0.33). However, the highest correlation was 
between CG and BL ( $r=0.91 ; \mathrm{P}<0.01)$ while the lowest was between CG and PR, SL and RT as well as TL and RT ( $\mathrm{r}=-0.01 ; \mathrm{P}>0.05$, respectively). At the finishing stage, strong relationship was observed between BW and body parameters such as CD (0.93), CG (0.92), BL (0.88), WH (0.86) and RH (0.83). RT and TL $(r=0.28 ; \mathrm{P}<0.05)$, PR and SL $(r=0.42 ; \mathrm{P}<0.01)$ and $\mathrm{PR}$ and TL $(\mathrm{r}=0.32 ; \mathrm{P}<0.05)$ were positively and significantly correlated. However, the relationship between WH and RH was strongest $(\mathrm{r}=$ $0.95 ; \mathrm{P}<0.01)$ while the weakest was between $\mathrm{WH}$ and $\mathrm{PR}(\mathrm{r}=0.03 ; \mathrm{P}>0.05)$.

Table 3. Growth stage and sex interaction effect on body weight, conformation and heat tolerance traits of pigs

\begin{tabular}{|c|c|c|c|c|c|c|c|}
\hline \multicolumn{3}{|c|}{ Piglets } & \multicolumn{2}{|l|}{ Growers } & \multicolumn{2}{|l|}{ Finishers } & \multirow{2}{*}{$\begin{array}{l}\text { P- } \\
\text { value }\end{array}$} \\
\hline Parameters & Female & Male & Female & Male & Female & Male & \\
\hline BW & $5.80 \pm 1.09^{\mathrm{a}}$ & $7.39 \pm 1.09^{\mathrm{a}}$ & $11.39 \pm 0.77^{\mathrm{a}}$ & $13.30 \pm 0.77^{\mathrm{a}}$ & $22.17 \pm 0.77^{b}$ & $29.83 \pm 0.77^{a}$ & 0.001 \\
\hline BL & $22.20 \pm 1.28^{\mathrm{a}}$ & $22.70 \pm 1.28^{\mathrm{a}}$ & $28.93 \pm 0.91^{\mathrm{a}}$ & $30.60 \pm 0.91^{\mathrm{a}}$ & $38.90 \pm 0.91^{\mathrm{b}}$ & $47.63 \pm 0.91^{\mathrm{a}}$ & 0.001 \\
\hline $\mathrm{CG}$ & $17.00 \pm 1.46^{\mathrm{a}}$ & $18.76 \pm 1.46^{\mathrm{a}}$ & $26.20 \pm 1.03^{\mathrm{a}}$ & $27.57 \pm 1.03^{\mathrm{a}}$ & $41.43 \pm 1.03^{b}$ & $51.37 \pm 1.03^{\mathrm{a}}$ & 0.001 \\
\hline $\mathrm{CD}$ & $7.40 \pm 0.75^{\mathrm{a}}$ & $8.63 \pm 0.75^{\mathrm{a}}$ & $14.75 \pm 0.53^{b}$ & $16.39 \pm 0.53^{\mathrm{a}}$ & $22.63 \pm 0.53^{b}$ & $26.63 \pm 0.53^{a}$ & 0.036 \\
\hline WH & $19.20 \pm 0.66^{\mathrm{a}}$ & $20.76 \pm 0.66^{a}$ & $28.50 \pm 0.47^{\mathrm{a}}$ & $29.53 \pm 0.47^{\mathrm{a}}$ & $39.50 \pm 0.47^{b}$ & $43.37 \pm 0.47^{\mathrm{a}}$ & 0.008 \\
\hline RH & $20.20 \pm 0.72^{\mathrm{a}}$ & $21.10 \pm 0.72^{\mathrm{a}}$ & $29.53 \pm 0.51^{\mathrm{a}}$ & $30.60 \pm 0.51^{\mathrm{a}}$ & $41.18 \pm 0.51^{b}$ & $45.13 \pm 0.51^{\mathrm{a}}$ & 0.007 \\
\hline $\mathrm{EL}$ & $2.63 \pm 0.29^{a}$ & $3.10 \pm 0.29^{a}$ & $7.29 \pm 0.20^{\mathrm{a}}$ & $7.90 \pm 0.20^{\mathrm{a}}$ & $10.53 \pm 0.20^{\mathrm{a}}$ & $11.10 \pm 0.20^{\mathrm{a}}$ & 0.956 \\
\hline SL & $3.40 \pm 0.45^{\mathrm{a}}$ & $4.23 \pm 0.45^{\mathrm{a}}$ & $8.33 \pm 0.32^{\mathrm{a}}$ & $8.87 \pm 0.32^{\mathrm{a}}$ & $11.60 \pm 0.32^{\mathrm{a}}$ & $12.64 \pm 0.32^{\mathrm{a}}$ & 0.725 \\
\hline $\mathrm{TL}$ & $3.54 \pm 0.48^{\mathrm{a}}$ & $3.94 \pm 0.48^{\mathrm{a}}$ & $8.10 \pm 0.34^{\mathrm{a}}$ & $8.18 \pm 0.34^{\mathrm{a}}$ & $10.67 \pm 0.34^{\mathrm{a}}$ & $10.73 \pm 0.34^{\mathrm{a}}$ & 0.915 \\
\hline RT & $39.61 \pm 0.75^{\mathrm{a}}$ & $39.02 \pm 0.75^{\mathrm{a}}$ & $38.88 \pm 0.53^{\mathrm{a}}$ & $38.06 \pm 0.53^{\mathrm{a}}$ & $37.92 \pm 0.53^{\mathrm{a}}$ & $37.93 \pm 0.53^{\mathrm{a}}$ & 0.718 \\
\hline PR & $77.47 \pm 2.36^{\mathrm{a}}$ & $80.00 \pm 2.36^{\mathrm{a}}$ & $75.80 \pm 1.67^{\mathrm{a}}$ & $74.83 \pm 1.67^{\mathrm{a}}$ & $79.27 \pm 1.67^{\mathrm{a}}$ & $80.60 \pm 1.67^{\mathrm{a}}$ & 0.649 \\
\hline RR & $39.13 \pm 1.06^{\mathrm{a}}$ & $34.40 \pm 1.06^{\mathrm{b}}$ & $39.13 \pm 0.75^{\mathrm{a}}$ & $40.67 \pm 0.75^{\mathrm{a}}$ & $39.63 \pm 0.75^{\mathrm{a}}$ & $39.33 \pm 0.75^{\mathrm{a}}$ & 0.004 \\
\hline
\end{tabular}

$B W=$ body weight; $B L=$ body length; $C G=$ chest girth; $C D=$ chest depth; $W H=$ withers height; $R H=$ rump height; $E L=$ ear length; $S L=$ snout length, $T L=$ tail length, $R T=$ rectal temperature; $P R=$ pulse rate; $R R=$ respiratory rate ${ }^{a b}$ Means within rows carrying the same superscripts do not differ significantly $(P>0.05)$ for all interactions

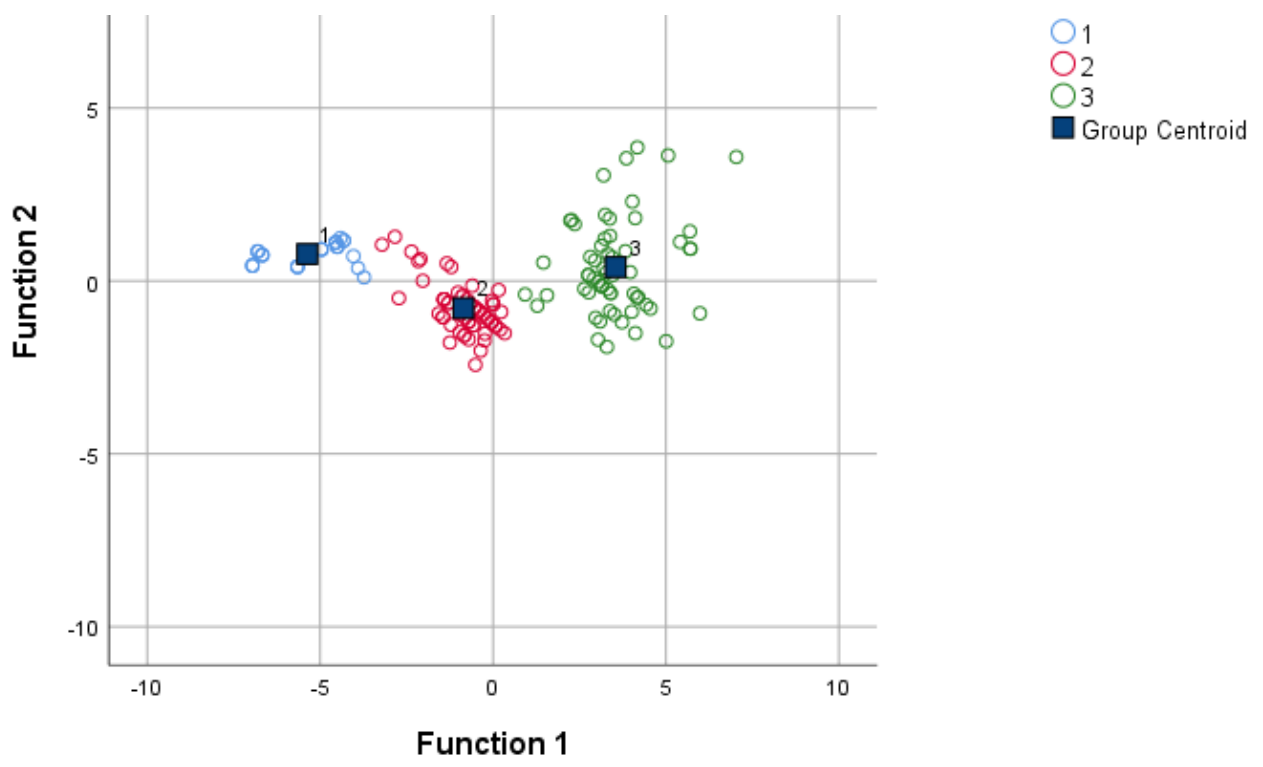

Figure 2. Canonical discriminant function showing the distribution among the three pig categories. $1=$ piglets; $2=$ growers; $3=$ finishers

Considering the low Anti-image correlations (partial correlations) obtained, there existed true factors in the data sets. This was strengthened by the high value (0.82) of Kaiser-Meyer- Olkin (KMO) measure of sampling adequacy, which revealed the proportion of the variance in $\mathrm{BW}$, conformation traits and heat 
Table 4. Phenotypic correlations of body weight, conformation and heat tolerance traits of pigs

\begin{tabular}{|c|c|c|c|c|c|c|c|c|c|c|c|c|c|}
\hline Traits & BW & $\mathrm{BL}$ & $\mathrm{CG}$ & $\mathrm{CD}$ & WH & $\mathrm{RH}$ & $\mathrm{EL}$ & $\mathrm{SL}$ & $\mathrm{TL}$ & $\mathrm{RT}$ & PR & RR & \\
\hline $\mathrm{BW}$ & & $0.80^{* *}$ & $0.92^{* *}$ & $0.91^{* *}$ & $0.93^{* *}$ & $0.88^{* *}$ & $0.85^{* *}$ & $0.89^{* *}$ & $0.88^{* *}$ & $-0.66^{* *}$ & 0.07 & -0.11 & \\
\hline $\mathrm{BL}$ & $0.77^{* *}$ & & $0.83^{* *}$ & $0.90^{* *}$ & $0.93^{* *}$ & $0.94^{* *}$ & $0.84^{* *}$ & $0.84^{* *}$ & $0.89^{* *}$ & $0.90^{* *}$ & $-0.68^{* *}$ & -0.16 & .157 \\
\hline $\mathrm{CG}$ & $0.72^{* *}$ & $0.91^{* *}$ & & $0.95^{* *}$ & $0.96^{* *}$ & $0.94^{* *}$ & $0.91^{* *}$ & $0.85^{* *}$ & $0.95^{* *}$ & $0.95^{* *}$ & $-0.67^{* *}$ & 0.02 & .080 \\
\hline $\mathrm{CD}$ & $0.58^{* *}$ & $0.42^{* *}$ & $0.44^{* *}$ & & $0.96^{* *}$ & $0.94^{* *}$ & $0.88^{* *}$ & $0.92^{* *}$ & $0.94^{* *}$ & $-0.67^{* *}$ & -0.07 & 0.05 & \\
\hline WH & $0.80^{* *}$ & $0.71^{* *}$ & $0.74^{* *}$ & $0.62^{* *}$ & & $0.98^{* *}$ & $0.89^{* *}$ & $0.88^{* *}$ & $0.93^{* *}$ & $-0.72^{* *}$ & -0.07 & 0.17 & \\
\hline $\mathrm{RH}$ & $0.66^{* *}$ & $0.61^{* * *}$ & $0.62^{* *}$ & $0.53^{* *}$ & $0.82^{* *}$ & & $0.83^{* *}$ & $0.80^{* *}$ & $0.90^{* *}$ & $-0.67^{* * *}$ & -0.14 & 0.26 & \\
\hline$\overline{E L}$ & $0.68^{* *}$ & $0.53^{* *}$ & $0.58^{* *}$ & $0.70^{* *}$ & $0.74^{* *}$ & $0.69^{* *}$ & & $0.91^{* *}$ & $0.98^{* *}$ & $-0.71^{* *}$ & 0.04 & -0.11 & \\
\hline$\overline{\mathrm{SL}}$ & $0.37^{* *}$ & $0.40^{* * *}$ & $0.37^{* *}$ & $0.57^{* *}$ & $0.46^{* *}$ & $0.52^{* * *}$ & $0.75^{\text {** }}$ & & $0.92^{* *}$ & $-0.70^{* * *}$ & -0.02 & -0.17 & \\
\hline $\mathrm{TL}$ & $0.26^{*}$ & $0.32^{*}$ & $0.26^{* *}$ & $0.60^{* *}$ & $0.35^{* *}$ & $0.42^{* * *}$ & $0.60^{* *}$ & $0.88^{* *}$ & & $-0.66^{* *}$ & 0.02 & -0.05 & \\
\hline$\overline{\mathrm{RT}}$ & $0.26^{*}$ & $0.34^{* *}$ & $0.41^{* * *}$ & 0.13 & 0.24 & 0.13 & 0.09 & -0.01 & -0.01 & & $0.42^{*}$ & 0.03 & \\
\hline PR & -0.05 & 0.05 & -0.01 & $-0.65^{* *}$ & -0.19 & -0.19 & $-0.41^{* *}$ & $-0.48^{* *}$ & $-0.58^{* *}$ & 0.05 & & -0.36 & \\
\hline$\overline{\mathrm{RR}}$ & 0.14 & -0.003 & 0.10 & $0.33^{* *}$ & 0.16 & 0.03 & 0.09 & -0.06 & 0.04 & -0.02 & -0.16 & & \\
\hline \multicolumn{14}{|l|}{$\overline{\mathrm{BW}}$} \\
\hline $\mathrm{BL}$ & $0.88^{* *}$ & & & & & & & & & & & & \\
\hline$\overline{\mathrm{CG}}$ & $0.92^{* *}$ & 0.88 & & & & & & & & & & & \\
\hline $\mathrm{CD}$ & $0.93^{* *}$ & $0.87^{* *}$ & 0.85 & & & & & & & & & & \\
\hline $\mathrm{WH}$ & $0.86^{* *}$ & $0.80^{* * *}$ & $0.76^{* *}$ & $0.83^{* *}$ & & & & & & & & & \\
\hline $\mathrm{RH}$ & $0.83^{* *}$ & $0.79^{* * *}$ & $0.76^{* *}$ & $0.80^{* * *}$ & $0.95^{* *}$ & & & & & & & & \\
\hline $\mathrm{EL}$ & $0.35^{* *}$ & $0.36^{\text {** }}$ & $0.29^{*}$ & $0.41^{* * *}$ & $0.47^{* *}$ & $0.49^{* * *}$ & & & & & & & \\
\hline SL & $0.34^{* *}$ & $0.52^{* *}$ & $0.33^{* *}$ & $0.40^{* * *}$ & $0.50^{* *}$ & $0.47^{* *}$ & $0.55^{* *}$ & & & & & & \\
\hline TL & $0.42^{* *}$ & $0.50^{* *}$ & $0.40^{* *}$ & $0.44^{* * *}$ & $0.55^{* *}$ & 0.59 & $0.62^{* *}$ & $0.67^{* *}$ & & & & & \\
\hline$\overline{\mathrm{RT}}$ & 0.09 & 0.16 & 0.12 & 0.17 & 0.15 & 0.14 & 0.18 & 0.15 & $0.28^{*}$ & & & & \\
\hline$\overline{\mathrm{PR}}$ & -0.11 & 0.16 & 0.10 & -0.11 & 0.03 & 0.10 & 0.10 & $0.42^{* * *}$ & $0.32^{*}$ & -0.05 & & & \\
\hline RR & 0.25 & 0.09 & 0.21 & 0.19 & 0.06 & 0.10 & 0.23 & -0.11 & 0.20 & 0.19 & -0.18 & & \\
\hline
\end{tabular}

BW=body weight; BL=bodylength; $C G=$ chest girth; $C D=$ chest depth; WH= withers height; RH= rump height $;$ EL=ear length; SL= sn out length, $T L=$ tail length, $R T=$ rectal temperature; $P R=$ pulse rate; $R R=$ respiratory rate ****Significant at $P<0.05$ and $P<0.01$, respectively

$1^{\text {st }}$ Upper diagonal= piglets; $2^{\text {nd }}$ Upper diagonal= growers; Lower diagonal $=$ finishers 
tolerance traits caused by the underlying factor. The overall significance of the correlation matrices tested with Bartlett's Test of Sphericity for the body parameters (chi-square $=704.657$; P < 0.01) supported the validity of the principal component analysis of the data sets. Principal components, eigenvalues and percentage of variance explained by components are shown in Table 5 and Figure 1, respectively. Based on the criterion of the eigenvalues-greater-than-one rule proposed by Kaiser, three principal components (PC1, PC2 and PC3) were retained. The three components had eigenvalues of 5.340, 2.353 and 1.565, respectively and explained about $77 \%$ of the generalized variance.

Based on Wilks' Lambda (0.056-0.105) and F statistics (155.375-625.864) (Table 6), withers height, ear length and body weight were the significant $(\mathrm{P}<0.001)$ parameters of importance to separate piglets, growers and finishers (Figure 2).

The prediction of group membership of piglets, growers and finishers is shown in Table 7. The classification results showed that $100 \%$ of piglets, $96.7 \%$ of growers and $96.7 \%$ of finishers were correctly assigned to their distinct groups. The three respective percentage values were $97.3 \%$ cross-validated.

The stepwise models predicting BW from linear body measurements are shown in Table 8. For piglets, withers height solely accounted for about $86 \%$ of the variation in body weight. The inclusion of body length in the model increased the proportion of the explained variance to about $88 \%$. The accuracy of the model was further improved $\left(\mathrm{R}^{2}=90.8 \%\right)$ when snout length was added to the equation. In growers, withers height alone contributed to $63.2 \%$ of the variation in body weight. The best prediction equation $\left(\mathrm{R}^{2}=0.722\right)$ was obtained when it was combined with body length. However, the highest single contributor $\left(R^{2}=0.869\right)$ to the variation in body weight of finishers was chest depth. However, the proportion of the explained variance progressively increased to $94.2 \%$ when chest girth, withers height and snout length were added to the model.

Table 5. Principal components, eigenvalues and percentage of variance explained by components (\% VCP) and communalities

\begin{tabular}{lllll}
\hline & PC1 & PC2 & PC3 & Communality \\
\hline Body weight & 0.976 & 0.004 & 0.139 & 0.971 \\
\hline Body length & 0.896 & 0.262 & 0.031 & 0.872 \\
\hline Chest girth & 0.918 & 0.080 & 0.062 & 0.853 \\
\hline Chest depth & 0.933 & 0.071 & 0.180 & 0.908 \\
\hline Withers height & 0.886 & 0.274 & 0.098 & 0.869 \\
\hline Rump height & 0.863 & 0.307 & 0.116 & 0.851 \\
\hline Ear length & 0.289 & 0.561 & 0.488 & 0.636 \\
\hline Snout length & 0.326 & 0.824 & 0.046 & 0.788 \\
\hline Tail length & 0.363 & 0.714 & 0.392 & 0.796 \\
\hline Rectal temperature & 0.027 & 0.155 & 0.644 & 0.440 \\
\hline Pulse rate & -0.083 & 0.728 & -0.322 & 0.641 \\
\hline Respiratory rate & 0.117 & -0.214 & 0.757 & 0.632 \\
\hline Eigenvalues & 5.340 & 2.353 & 1.565 & \\
\hline Percentage VCP & 44.498 & 19.606 & 13.038 & \\
\hline Percentage VCP Cumulative & 44.498 & 64.104 & 77.141 & \\
\hline
\end{tabular}

Table 6. Variables of importance in separating piglets, growers and finishers

\begin{tabular}{llccccc}
\hline Parameters & Wilks' Lambda & df1 & df2 & df3 & F-value & Significance \\
\hline Withers height & 0.105 & 1 & 2 & 147.000 & 625.864 & 0.001 \\
\hline Ear length & 0.066 & 2 & 2 & 147.000 & 211.327 & 0.001 \\
\hline Body weight & 0.056 & 3 & 2 & 147.000 & 155.375 & 0.001 \\
\hline
\end{tabular}

Table 7. Assignment of the three pig populations into groups 


\begin{tabular}{llllll}
\hline & Growth stage & Piglets & Growers & Finishers & Total \\
\hline Original count & Piglets & 30 & 0 & 0 & 30 \\
\hline & Growers & 2 & 58 & 0 & 60 \\
\hline$\%$ & Finishers & 0 & 2 & 58 & 60 \\
\hline & Piglets & 100.0 & 0.0 & 0.0 & 100.0 \\
\hline & Growers & 3.3 & 96.7 & .0 & 100.0 \\
\hline Cross-validated count & Finishers & 0.0 & 3.3 & 96.7 & 100.0 \\
\hline & Piglets & 30 & 0 & 0 & 30 \\
\hline$\%$ & Growers & 2 & 58 & 0 & 60 \\
\hline & Adults & 0 & 2 & 58 & 60 \\
\hline & Piglets & 100.0 & 0.0 & 0.0 & 100.0 \\
\hline & Growers & 3.3 & 96.7 & .0 & 100.0 \\
\hline
\end{tabular}

$97.3 \%$ of original grouped cases correctly classified.

97.3\% of cross-validated grouped cases correctly classified.

Table 8. Stepwise multiple regression of body weight on body measurements of pigs

\begin{tabular}{|c|c|c|c|c|}
\hline Model & Significance & $\mathrm{R}^{2}$ & Adjusted $\mathrm{R}^{2}$ & $\overline{\mathrm{RM}}$ \\
\hline \multicolumn{5}{|l|}{ Piglets } \\
\hline $1 . \mathrm{BW}=-3.407+0.501 \mathrm{WH}$ & $\mathrm{P}<0.01$ & 0.855 & 0.850 & $\overline{0.52}$ \\
\hline $2 . \mathrm{BW}=-1.656+0.728 \mathrm{WH}-0.280 \mathrm{BL}$ & $\mathrm{P}<0.01$ & 0.883 & 0.875 & $\overline{0.48}$ \\
\hline $3 . \mathrm{BW}=-0.237+0.571 \mathrm{WH}-0.319 \mathrm{BL}+0.675 \mathrm{SL}$ & $\mathrm{P}<0.01$ & 0.917 & 0.908 & $\overline{0.41}$ \\
\hline \multicolumn{5}{|l|}{ Growers } \\
\hline 1. $\mathrm{BW}=-20.160+1.120 \mathrm{WH}$ & $\mathrm{P}<0.01$ & 0.632 & 0.626 & $1.40^{\prime}$ \\
\hline 2. $\mathrm{BW}=-18.559+0.698 \mathrm{WH}+0.358 \mathrm{BL}$ & $\mathrm{P}<0.01$ & 0.722 & 0.713 & 1.23 \\
\hline \multicolumn{5}{|l|}{ Finishers } \\
\hline 1. $\mathrm{BW}=-20.384+1.883 \mathrm{CD}$ & $\mathrm{P}<0.01$ & 0.869 & 0.867 & 2.66 \\
\hline 2. $\mathrm{BW}=-16.877+1.108 \mathrm{CD}+0.336 \mathrm{CG}$ & $\mathrm{P}<0.01$ & 0.924 & 0.922 & $\overline{2.04}$ \\
\hline 3. $\mathrm{BW}=-24.656+0.853 \mathrm{CD}+0.305 \mathrm{CG}+0.374 \mathrm{WH}$ & $\mathrm{P}<0.01$ & 0.936 & 0.933 & 1.89 \\
\hline 4. $\mathrm{BW}=-24.683+0.865 \mathrm{CD}+0.295 \mathrm{CG}+0.469 \mathrm{WH} 0.309 \mathrm{SL}$ & $\mathrm{P}<0.01$ & 0.942 & 0.938 & 1.82 \\
\hline
\end{tabular}

$R^{2}=$ coefficient of determination; $R M S E=$ root mean square error

\section{Discussion}

The higher values of finishers are consistent with the normal growth trajectories where adults are bigger in size than the young ones. This is consistent with the earlier submission that age greatly influence body weight and conformation traits of animals, and that growth follows a general pattern till maturity stage (Yakubu et al., 2011). The body weight values of the three pig categories of the present study are comparable to the range of 9 $32 \mathrm{~kg}$ (female) and 8 - $37 \mathrm{~kg}$ (male) in Nigerian indigenous pigs (NIP) and crossbred pigs (CBP) reported by Adeola et al. (2013) in a humid tropical zone of Nigeria. The body weight range $(5.80-7.39 \mathrm{~kg})$ of the Nigerian indigenous piglets is also comparable to the $6.8 \mathrm{~kg}$ of low performing population, but less than the $12.2 \mathrm{~kg}$ of high performing population 6-week-old Hypor Libra' piglets in Netherlands (Paredes et al., 2014). The average body weight of the Nigerian indigenous piglets appeared lower than the $8.40 \pm 2.04 \mathrm{~kg}$ found in purebred Berkshire pigs reared in a hoop structure (Park and Oh, 2018). The variation may be attributed to genotype and environmental factors. However, the small body size of the Nigerian indigenous piglets may be an adaptive strategy to withstand the prevailing harsh environmental conditions.

Sexual dimorphism is a widespread phenomenon among animal groups (Yakubu, 2011; Bozkurt and Can, 2021). The most likely traits to exhibit sexual dimorphism are body size and shape. At inter-population level especially with size and most conformation traits, sexual dimorphism in the present study favoured male animals. The differential rate and duration of growth may be responsible for the present observations. This is in 
consonance with the report of Baeza et al. (2001) that between-sex differential hormonal action may invariably lead to differential growth rates in males and females. The present differences may also be attributed to strong sexual selection favouring large males whereas the females were subjected to weak fecundity selection. According to Salogni et al. (2018), high male-biased dimorphism in body size is as a result of the physical competition among males in order to have access to breeding females; hence the possession of large size can assist greatly in winning contests or allow the extension of a male's breeding tenure. It has been postulated that genes for important economic traits may be differently expressed in males and females (van der Heide et al., 2016; Fairbairn, 2016). This was buttressed by the submission of Raidan et al. (2019) that in the presence of genotype-by-sex interactions, selection for traits in each sex may result in high rates of genetic improvement. However, if animals are to be identified based on highest breeding value, the data for females and males may be considered a single trait (Raidan et al., 2019). The varying growth stage by sex interaction effect permits the different ranking of piglets, growers and finishers under the two sexes. The respiratory rate and rectal temperature of the present study were lower than the values of $43.75-72.12$ breaths $/ \mathrm{min}$. and $39.05{ }^{\circ} \mathrm{C}-39.57$ ${ }^{\circ} \mathrm{C}$ obtained in pigs reared in India (Pathak et al., 2018). The differential values could be as a result of varying genetic groups, weather conditions and management practices.

The high correlation of rectal temperature with body weight and conformation traits in piglets is an indication that this parameter could be a good indicator of welfare and comfort (Zaake, 2018). According to Zaake (2018), rectal temperature is one of the key heat stress parameters, which was found to be associated with chest girth in pigs. Achieving optimal development of thermoregulation is a challenge that piglets must confront to successfully adapt to extrauterine life (Villanueva-García et al., 2021), especially in the hot-dry season in a tropical guinea savannah of Nigeria (Zakari et al., 2021). The high positive correlations observed in the present study suggest that selection for a trait may lead to a correlated response in the other trait. Strong association between body weight and other parameters could lead to its prediction from linear body measurements. The present correlation values are comparable to the values reported by Adeola et al. (2013) in indigenous pigs of southwestern Nigeria. In another study on pigs under tropical conditions, Oluwole et al. (2014) found high correlations between body weight and morphometric traits.

The relevance of $\mathrm{PC}$ as a multivariate statistical tool was evidenced in the reduction of large number of explanatory variables into components that gave a better description of size and shape. In the present study, twelve explanatory variables have been reduced to three components. The three principal components (PC 1, PC 2 and PC 3) obtained could be useful in evaluating animals for breeding and selection purposes. Since correlation between principal components is zero, the selection of animals for any principal component will not cause a correlated response in terms of other principal components (Pinto et al., 2006; Yakubu and Ari, 2018). In this wise and across the three growth stages in this study, selecting for body weight will positively affect the selection of body length, chest girth, chest depth, withers height and rump height. Also, selecting for ear length, snout length, tail length (PC2) could invariably lead to pulse rate selection. The ability of the discriminant function to successfully classify the three growth stages can be useful in making general management decisions especially in smallholder farms.

Under the resource poor low-input setting, farms do not have complete restraint and handling systems, and few have animal scales to determine body weights. Therefore, equations to estimate body weight from other body measurements are needed (Heinrichs et al., 1992). Withers height was found as a very good parameter to estimate body weight in the present study. This might not be unconnected with the fact that wither height is a skeletal parameter that is not influenced by body condition. It is important that growth recommendations be based on body weight within the parameters of desired skeletal growth (Heinrichs et al., 1992). This is because large body weight during early life without corresponding skeletal growth may result in impaired mammary development and reduced milk production (Sejrsen et al., 1982). The present findings on body weight and body dimensions of pigs could aid the design of modern housing structures and equipment as postulated by Smith and Ramirez (2021). 


\section{Conclusion}

The indigenous pigs in Plateau State Nigeria were of small body weights and morphometric traits, which could be part of the animals' adaptation for survival under the low-inputs tropical environment. High correlations were observed between body weight and most linear body measurements. The resultant three principal components could aid in selection and breeding programmes of the pigs. Withers height, ear length and body weight were sufficient to assign the pigs into their appropriate growth stages. Withers height was the best single parameter to predict body weight. The phenotypic Information obtained in this study may be exploited in subsequent managerial decisions to improve pig production in the study area. There is need for future study on molecular characterization to better understand the genome of the pigs.

\section{Acknowledgments}

All the farmers and village heads involved in the study are highly appreciated.

\section{Funding}

This study did not receive any external financial support.

\section{Data availability}

Availability of data will be based on request.

\section{Authors' Contributions}

AY and GLD designed the work. The field work was carried out by GLD. Data analys is was carried out by AY. The first draft was written by GLD and proofread by AY and JH. The final draft was approved by all authors.

\section{Conflict of Interest}

The authors declare that there is no conflict of interest.

\section{References}

Abed F. Bachir-Bouiadjra B. Dahloum L. Yakubu A. Haddad A. Homrani A 2021. Procruste analysis of forewing shape in two endemic honeybee subspecies Apis mellifera intermissa and Apis mellifera sahariensis from the Northwest of Algeria. Biodiversitas Journal of Biological Diversity 22: 154-164. DOI: 10.13057/biodiv/d220121.

Adeola AC. Oseni SO. Omitogun OG 2013. Morphological characterization of indigenous and crossbred pigs in rural and peri-urban areas of southwestern Nigeria. Open Journal of Animal Sciences 3: 230-235.

Al Ard Khanji MS. Llorente C. Falceto MV. Bonastre C. Mitjana O. Tejedor MT 2018. Using body measurements to estimate body weight in gilts. Canadian Journal of Animal Science 98: 362-367. dx.doi.org/10.1139/cjas-2016-0232

AU-IBAR 2015. Pictorial field guide for linear measurements of animal genetic resources. African Union InterAfrican Bureau for Animal Resources (AU-IBAR), Nairobi, Kenya. 47pp.

Baeza E. Williams N. Guemene D. Duclos MJ 2001. Sexual dimorphism for growth in Muscovy duck and changes in insulin-like growth factor I (IGF- I), growth hormone (GH) and triiodothyronine (T3) plasma levels. The Journal of Reproduction and Development 41: 173-179.

Belkhadem S. Tefiel 2. Belantar I. Chahbar M. Gaouar SBS 2019. Discriminant analysis on the morphometry of local goats breed in the western of Algeria. Genetics and Biodiversity Journal 3: 49-56

Bozkurt A. Can M 2021. Diversification of Sexual Size Dimorphism in Cyclops vicinus. Kahramanmaraş Sütçü Imam Üniversitesi Tarım ve Doğa Dergisi 24: 824-833. DOI: 10.18016/ksutarimdoga.vi.825506

B rown-Brandi TM. Nienaber JA. Xin H. Gates RS 2004. A literature review of swine heat production. In: Transactions of the ASAE 47:pp. 259-270. 
ESGPIP 2009. TECHNICAL BULLETIN No.23 Estimation of weight and age of sheep and goats. Ethiopia Sheep and Goat Productivity Improvement Program (ESGPIP). 14pp.

Fairbairn DJ 2016. Sexual Dimorphism. In: Encyclopedia of Evolutionary Biology (edt. Richard M. Kliman). ISBN 978-0-12-800426-5. Elsevier, Netherlands.

Fernandes AFA. Dórea JRR. Fitzge rald R. Herring W. Rosa GJM. 2019. A novel automated system to acquire biometric and morphological measurements and predict body weight of pigs via 3D computer vision. Journal of Animal Science 97: 496-508. doi: 10.1093/jas/sky418

Fraga AB. de Lima Silva F. Hongyu K. Da Silva Santos D. Murphy T. Lopes FB 2016. Multivariate analysis to evaluate genetic groups and production traits of crossbred Holstein x Zebu cows. Tropical Animal Health and Production 48:533 538.

Heinrichs J. Rogers GW. Cooper JB 1992. Predicting body weight and wither height in Holstein heifers using body measurements. Journal of Dairy Science 75: 3576-3581.

SPSS 2017. IBM Corp. Released 2017. IBM SPSS Statistics for Windows, Version 25.0 Armonk, NY: IBM Corp.

Kohler-Rollefson I 2000. Management of animal genetic diversity at community level. Management Agrobiodiversity in Rural areas. DeutecheGesellschafi fur Technishe Zusammenarbeit (GTZ)GmbH.Eschbom.ftp://flp.fao.-org/docrep/nonfao/lead/x6103e/x6103e00.pdf.

Mediouni RM. Said S. Ameur Ameur A. Gaouar SBS 2020. Phenotypic and morphometric diversity of Indigenous Turkey (Meleagris Gallopavo) from Wilaya of Tlemcen, Northwest of Algeria. Genetics and Biodiversity Journal 4: 1-10.

Meka Z II. Tadakeng Y. Meutchieye F. Fonteh F 2021. Biometric assessment of blackbelly sheep in Central Africa. Genetics and Biodiversity Journal 5: 139-153.

Morenikeji, OB. Ogunshola OJ. Adu OA. Aro SO. Chineke CA 2018. Genotype and sex effects on the performance characteristics of pigs. International Journal of Livestock Production 10: 127-134. doi: 10.5897/IJLP2015.0254

Mutua FK. De wey CE. Arimi SM. Schelling E. Ogara WO 2011. Prediction of live body weight using length and girth measurements for pigs in rural Western Kenya. Journal of Swine Health and Production 19:26-33.

National Bureau of Statistics (NBS) 2011. National Agriculture Sample Survey, Nigeria 2010-2011, Public Access Dataset, Accessed on October 18, 2016.

Oluwole AA. Tiamiyu AK. Olorungbounmi TO. Oladele-Bukola MO. Akintoye NA 2014. Pre-Weaning growth traits in Nigerian indigenous pig crossbreds. Agricultural Sciences 5: 891-896.

Panda S. Gaur GK. Chauhan A. Kar J. Mehrotra A. 2021. Accurate assessment of body weights using morphometric measurements in Landlly pigs. Tropical Animal Health and Production 53: 362. https $/ /$ doi.org/10.1007/s11250-021-02803-2

Pare des SP. Jansman AJM. Verstegen MWA. den Hartog LA. van Hees HMJ. Bolhuis JE. van Kempen TATG. Gerrits WJJ. 2014. Identifying the limitations for growth in low performing piglets from birth until 10 weeks of age. Animal 8: 923-930.

Park H-S. Oh S-H. 2018. Factors affecting growth and body dimensions of pigs reared in alternative production. Journal of Applied Animal Research 46:1, 587-592. DOI: 10.1080/09712119.2017.1366323

Pathak PK. Roychoudhury R. Saharia J. Borah MC. Dutta DJ. Bhuyan R. Kalita D. 2018. Impact of seasonal thermal stress on physiological and blood biochemical parameters in pigs under different dietary energy levels. Tropical Animal Health and Production 50(5): 1025-1032. doi: 10.1007/s11250-018-1526-6.

Pinto LFB. Dacker IU. De melo CMR. Ledur MC. Coutinho LL. 2006. Principal components analysis applied to performance and carcass traits in the chicken, Animal Research 55:419- 425. 
Raidan FSS. Porto-Neto LR. Reverter A. 2019. Across-sex genomic-assisted genetic correlations for sexinfluenced traits in Brahman cattle. Genetics Selection Evolution 51(1): 41. doi: 10.1186/s12711-019-04826.

Rege JEO. Mars hall. Notenbaen A. Ojango J. Mwai AO. 2011. Pro-poor animal improvement and bre eding What can science do Livestock Science 136: 15-28.

Smith BC. Ramirez BC. 2021. Dimensions of the Suckling Pig. Frontiers in Animal Science 2 31: DOI=10.3389/fanim.2021.694299.

Salogni E. Galimberti F. Sanvito S. Miller EH 2018. Male and female pups of the highly sexually dimorphic northern elephant seal (Mirounga angustirostris) differ slightly in body size. Canadian Journal of Zoology 97: 241-250. https $/ /$ doi.org/10.1139/cjz-2018-0220s

Schwabe AE. Hall SJG 1989. Dystocia in nine British breeds of cattle and its relationships to the dimensions of the dam and calf. Veterinary Record 125: 636-639.

Sejrsen K. Huber JT. Tucker HA. and Akers RM 1982. Influence of nutrition on mammary development in pre- and post-pubertal heifers. Journal of Dairy Science 65: 793.

Shi C. Zhang J. Teng G. 2020. Division of pig growth stages according to body component variation using computer vision. Pakistan Journal of Zoology 52: 1071-1078. DOI: https $/ /$ dx.doi.org/10.17582/journal.pjz/20191016031055

Silva Filha OL. Pimenta-Filho EC. Silva LPG. Pe reira WE. Oliveira RJF. Delgado JV. Sereno JRB 2010. Body morphometry of local pigs of Curimatau Paraibano. Multivariate analysis. Revista Computadorizada de Produccion Porcina 17: 197-202.

Sie we AT. Manjeli Y. Meutchie ye F. 2021. The Bankim Pigs: A native Cameroonian breed assessed by biometric features. Genetics and Biodiversity Journal 5 (1): 101-111.

Sobczuk D. Komosa M 2012. Morphological differentiation of Polish Arabian horses Multivariate analysis. Bulletin of the Veterinary Institute Pulawy 56: 623-629.

Sipos W. Wiener S. Entenfellner F. Sipos S 2013. Physiological changes of rectal temperature, pulse rate and respiratory rate of pigs at different ages including the critical peripartal period. Wiener Tierärztliche Monatsschrift - Veterinary Medicine Austria 100: 93-98.

Tabachnick B G. Fidell LS 2001. Using Multivariate Statistics. Allyn and Bacon, Boston, USA. 966 pp.

Van der Heide EM. Lourenco DA. Chen CY. Herring WO. Sapp RL. Moser DW. Tsuruta S. Mas uda Y. Ducro BJ. Misztal I 2016. Sexual dimorphism in livestock species selected for economically important traits. Journal of Animal Science 94: 3684-3692. doi: 10.2527/jas.2016-0393.

Ventura HT. Lopes PS. Peloso JV. Guimaraes SEF. Cameiro APS. Cameiro PLS 2012. Use of multivariate analysis to evaluate genetic groups of pigs for dry-cured ham production. Livestock Science 148: 214-220.

Villanue va-García D. Mota-Rojas D. Martínez-Burnes J. Olmos-Hernández A. Mora-Medina P. Salme rón C. Gómez J. Bos cato L. Gutiérrez-Pérez O. Cruz V. Reyes B. González-Lozano M 2021. Hypothermia in newly born piglets: Mechanisms of thermoregulation and pathophysiology of death. Journal of Animal Behaviour and Biometeorology 9: 2101. http://dx.doi.org/10.31893/jabb.21001.

Vohra V. Niranjan SK. Mishra AK. Jamuna V. Chopra A. Sharma N. Jeong DK 2015. Phenotypic characterization and multivariate analysis to explain body conformation in lesser known Buffalo (Bubalusbubalis) from North India. Asian Australasian Journal of Animal Science 28: 311-317.

Walugembe M. Nadiope G. Stock JD. Stalder KJ. Pezo D. Rothschild MF 2014. Prediction of live body weight using various body measurements in Ugandanvillage pigs. Livestock Research for Rural Development. Volume 26, Article 96. Retrieved November 5, 2016, from http://www.lrrd.org//rrd26/5/walu26096.html 
Yakubu A. Ari MM 2018. Principal component and discriminant analyses of body weight and conformation traits of Sasso, Kuroiler and indigenous Fulani chickens in Nigeria. Journal of Animal and Plant Sciences 28 (1): 46-55.

Yakubu A 2013. Principal component analysis of the conformation traits of Yankasa sheep. Biotechnology in Animal Husbandry 29 (1): 65-74.

Yakubu A 2011. Discriminant analysis of sexual dimorphism in morphological traits of African Muscovy ducks (Cairina moschata). Archivos de Zootecnia 60: 1115-1123. scielo.isciii.es/pdf/azoo/v60n232/art27.pdf

Yakubu A. Salako AE. Abdullah AR 2011. Varimax rotated principal component analysis of the zoometrical traits of Uda sheep. Archivos de Zootecnia, 60(231): 813-816. DOI: 10.4321/S0004-05922011000300069

Yakubu A. Salako AE. Lmumorin IG. Ige AO. Akinyemi MO 2010. Discriminant analysis of mor phometric differentiation in the West African Dwarf and Red Sokoto goats. South African Journal of Animal Science 40: 381-387.

Zaake P 2018. An assessment of heat stress status in pigs and adaptation options in lira district Uganda. Thesis (MSc. in Climate Change Adaptation). University of Nairobi. 76 p.

Zakari FO Akefe IO Uchendu C 2021. Comparison of diurnal rectal and body surface temperatures in large white piglets during the hot-dry season in a tropical Guinea savannah. Journal of Thermal Biology (in press). DOI: 10.1016/j.jtherbio.2021.102953.

Zhang J. Zhuang Y. Ji H. Teng G 2021. Pig weight and body size estimation using a multiple output regression convolutional neural network: A fast and fully automatic method. Sensors (Basel), 6;21(9): 3218. doi: 10.3390/s21093218. 